\title{
Dynamic Monte Carlo Simulation of an Amorphous Organic Device
}

\author{
Gregor Meller, Ling Li, Stefan Holzer, and Hans Kosina \\ *Institute for Microelectronics, TU Wien, 1040 Vienna, Austria \\ \{meller|ling.li|holzer|kosina\}@iue.tuwien.ac.at
}

\begin{abstract}
Upcoming organic devices like thin $1 \mathrm{~m}$ transistors or light emitting diodes require novel architectures and materials. In most cases, two main problems, namely poor carrier mobility in the bulk and elusive interface effects, are closely related due to contact dominated device behavior. A three-dimensional Kinetic Monte Carlo simulator has been developed mimicking simultaneously charge injection and propagation. This work deals with the representation of contact roughness by the means of the Gaussian Disorder Model.
\end{abstract}

\section{Introduction}

Hydrocarbons with alternating double or triple bonds exhibit dangling $\mathrm{sp}^{3}$-hybridized $\pi$ orbitals interfering constructively or destructively. In the former case the $\pi$ electron's charge density concentrates between the repulsive carbon cores, thereby screening their mutual electrostatic repulsion. These electrons are thus called bonding, $\pi$ electrons. Destructively interfering $\pi$ wavefunctions, on the other hand, concentrate the electron charge densities $a t$ the ionic cores, thus enhancing the cores' mutual Coulombic repulsion. These states are labeled as antibonding, $\pi^{*}$ orbitals [1]. The p-conductive $\pi$ as well as the n-conductive $\pi^{*}$ bands form branched wave functions spread across the $\sigma$ bound molecular skeleton. The degree of carrier delocalization along these bands determines the carrier mobilities and depends on the amount of disorder inherent in the system. A lack of long range order defeats overall molecular conjugation. In other words, the molecular polarization energies are locally too different for carrier delocalization across the whole molecular solid. If, however, the wave functions attached to contiguous $\pi$ conjugated entities like chromophores, molecules, or oligomers overlap suf ciently strong, thermally assisted tunneling might allow a random walk along the $\pi / \pi^{*}$ bands at room temperatures. The spectral gap separating the bonding from the antibonding orbitals, typically $\approx 3 \mathrm{eV}$, prohibits the thermal excitation of intrinsic carriers from the valence into the conduction band at room temperatures. The resulting lack of intrinsic carriers necessitates the injection of currents from either metal or polymer contacts. The remarkable strong in uence of the injection ef ciency on the device performance can be envisioned by the carrier concentration dependence of the mobility, which may vary by several orders of magnitude depending on the number of electrons injected [2]. The complex interplay between thermionic emission, tunneling, space-charge effects and trapping is mirrored in rapidly varying transport regimes [3]. 
Thus, neither the Richardson-Schottky formula nor the Fowler-Nordheim expression adequately describes the device behavior over the full operational range [4].

\section{Simulation}

Phonon assisted tunneling in the presence of a eld causes a biased random walk among localized $\pi$ and/or $\pi^{*}$ states characterized by size $a$, shape $\Psi(r)=e^{-r / a}$ and energies $\varepsilon$ [5]. We label the mutual distances $R_{i j}$, take the Miller-Abrahams rate composed of the attempt-to-escape frequency $v_{0}$, an overlap-dependent tunneling and a Boltzmannian single-phonon absorption rate [6],

$$
v_{i j}=v_{0} \exp \left[-\frac{2 R_{i j}}{a}\right] \times\left\{\begin{aligned}
\exp \left[-\frac{\varepsilon_{j}-\varepsilon_{i}}{k_{B} T}\right] & \varepsilon_{j}>\varepsilon_{i} \\
1 & \varepsilon_{j}<\varepsilon_{i}
\end{aligned}\right.
$$

and use the queue of events established by the long tailed distributions of waiting times

$$
\left\langle\tau^{i}\right\rangle=\left(\sum_{j \neq i} v_{i j}\right)^{-1}
$$

as trigger for a stochastic time evolution. The possibility to interpret this event-driven technique actually in a dynamic sense is a peculiarity of Kinetic Monte Carlo algorithms [7, 8]. The self-adjusting timesteps generate a continuous time random walk [9]. The complete algorithm is outlined in Fig. 1(a). Charging and discharging with fermions is considered, each level consists of one spin-up and one spin-down orbital capable of holding one electron. The energies are distributed according to the uncorrelated Gaussian Disorder Model [10]. Since the slowly migrating charges behave like resting

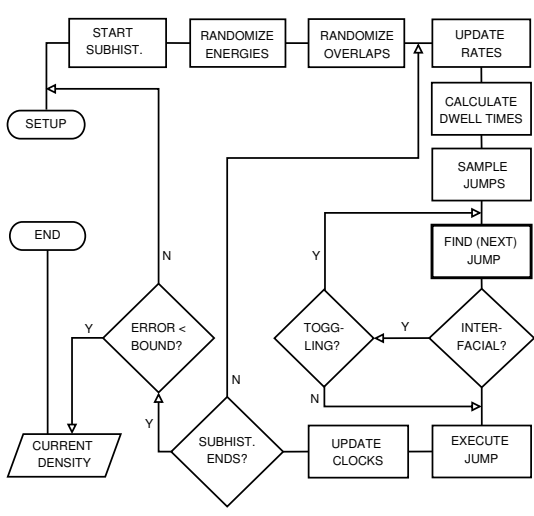

(a)

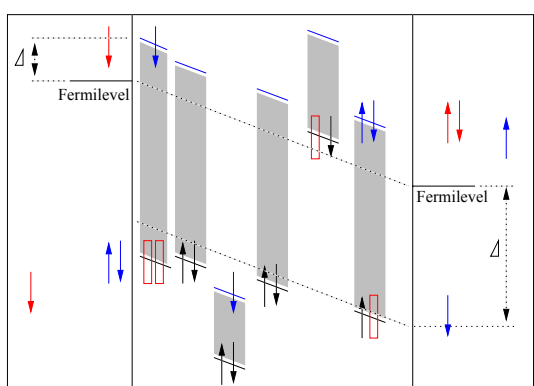

(b)

Figure 1: (a): The algorithm. All criteria stated in [17] are met, so the phase space traversal can be related to a Poisson process. In this work, toggling interface states were not suppressed. (b): The two-level site concept including $\mathrm{p}$ and n-type doping. The rectangles and arrows at the bottom levels stand for $\pi$ vacancies and electrons respectively. The upper levels mark pairs of $\pi^{*}$ orbitals. 
Coulomb centers [11], each electron's potential has to be considered in calculating the present space charge. Holes are modeled quite differently from electrons. We assume that the depletion of a bonding state exposes an immobile positive Coulomb center of charge $+e$ stemming from the otherwise sreened ionic background charge of the polymer backbone. Since each $\pi / \pi^{*}$ level consists of two $\pi / \pi^{*}$ states, a completely discharged $\pi$ level generates a potential of charge $+2 e$. Occupied antibonding states correspond to Coulomb centers of charge $-e$. Completely lled $\pi^{*}$ levels thus generate potentials of charge $-2 e$. All potentials are screened by the dielectric constant of the material, $\varepsilon_{r}$, whose small value enhances the in uence of the image force potentials on the injection barriers and thus on the whole device performance [12]. Locally varying Schottky effects due to very rough electrodes cause interfacial energetic disorder [14], whereas moderate roughness does not affect carrier injection signi cantly [15]. In Bässler's model, contact roughness and the like have to be mastered by the means of Gaussians. Fig. 1(b) illustrates the interplay of mobile and immobile charges and their mirror images as taken into account by the computer program. The mirror charges of virtual carriers outside the simulation domain are also considered. Following [12], injection is modeled as a Miller-Abrahams hop from the metal into the polymer. The departure level accounts for the fact that metal electrons close to the surface are captured in a potential well characterized by the electrode surface's work function [13].

\section{Results and Discussion}

The undoped p-conductive device Gold/Zinc Phthalocyanine/Gold is simulated. The measured band structure [16] is summarized in Fig. 2(a). The lattice constant $(0.6 \mathrm{~nm})$, the maximal jump distance $(1.2 \mathrm{~nm}), T=300 \mathrm{~K}, \varepsilon_{r}=3.0$, and $v_{0}=1.0 \times 10^{13} \mathrm{~s}^{-1}$ have been assumed positionally independent. The interfacial wave function decay $1 / a=2.08 \times 10^{10} \mathrm{~m}^{-1}$ exceeded that in the bulk $\left(5.553 \times 10^{9} \mathrm{~m}^{-1}\right)$. Fig. 2 (b) de-

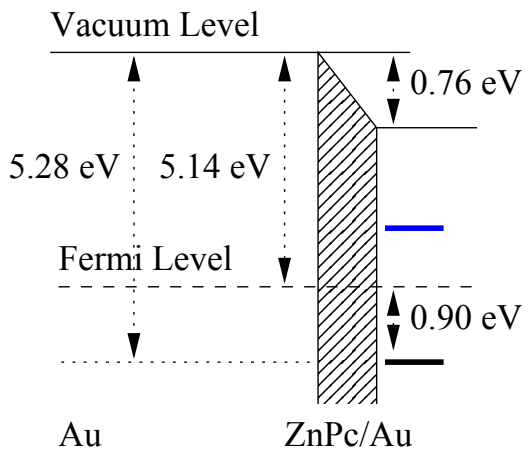

(a)

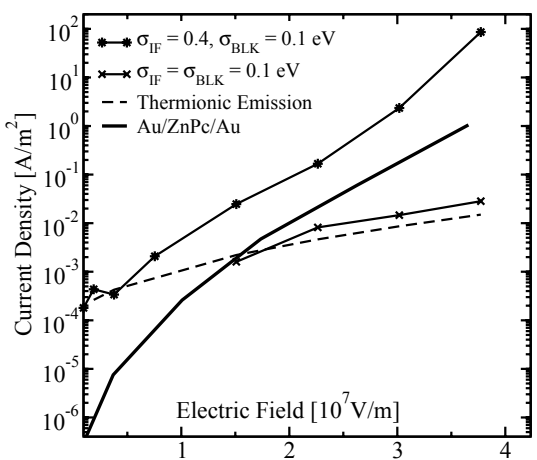

(b)

Figure 2: (a): The interface dipole layer, work functions and ionization energies as measured by [16]. (b): the width $\sigma$ of the Gaussian density of states measures the molecular disorder. The bold curve depicts the measured current, the dashed curves the current as predicted by the Richardson-Schottky formula. 
picts the interplay between interface and bulk disorder. Following the ndings of [14] the interfacial energetic disorder $\sigma_{\mathrm{IF}}=0.4 \mathrm{eV}$ outranges that of the bulk by a factor of 4 . The simulation shows good coincidence with the measurement and thus injection limited behavior. Setting $\sigma_{\mathrm{IF}}=0.1 \mathrm{eV}$ reduces the number of injection facilitating tail states and the current approaches the thermionic one.

\section{Acknowledgements}

This work has been supported by the Austrian Science Fund, grant P16862-N02.

\section{References}

[1] H. Kuhn, H.D. Försterling, "Principles of Physical Chemistry", ISBN: 0-471-95902-2, John Wiley \& Sons, LTD, pp. 169-183, 2000.

[2] C. Tanase, E.J. Meijer, P.W.M. Blom, D.M.de Leeuw, "Uni cation of the Hole Transport in Polymeric Field-effect Transistors and Light Emitting Diodes", Physical Review Letters, 91 (21), pp. 216601-1-216601-4.

[3] Schwoerer, M. and Wolf, H.C., Organische Molekulare Festkörper, pp. 235-236, Wiley$\mathrm{VCH},(2005)$.

[4] J.C. Scott, and G.G. Malliaras, "Charge Injection and Recombination at the Metal-Organic Interface", Chem. Phys. Lett, vol. 299, no. 2, pp. 115-119, 1999.

[5] G. Horowitz, "Physics of Organic Field-Effect Transistors", Semiconducting Polymers, Wiley-VCH, pp. 463-514, 2000.

[6] A. Miller, and E. Abrahams, "Impurity Conduction at Low Concentrations", Phys. Rev. 120, pp. $745-755,1960$

[7] A.F. Voter, "Introduction to the Kinetic Monte Carlo Method", in "Radiation Effects in Solids", edited by K.E. Sickafus and E.A. Kotomin, Springer, NATO Publishing Unit Dortrecht, 2005, preprint.

[8] D. Johnson, "Kinetic Monte Carlo: Bare Bones and a Little Flesh", Lecture given at the Summer School on Computational Materials Science, University of Illinois, Urbana-Champaign, 2001. http://www.mcc.uiuc.edu/summerschool/2001/

[9] J. Klafter, G. Zumofen, "Probability Distributions for Continuous-Time Random Walks with Long Tails", J. Phys. Chem, 98, pp. 7366-7370, 1994.

[10] H. Bässler, "Charge Transport in Disordered Organic Photoconductors", Phys.Stat.Sol. (b), 175, pp. 15-56, 15, 1993.

[11] H. Houili, L. Zuppiroli, "Investigation of the Charge Transport through Disordered Organic Molecular Heterojunctions", J. Appl. Phys., 100, 1, pp. 1-12., 2006.

[12] U. Wolf, V.I. Arkhipov, and H. Bässler, "Current Injection from a Metal into a Disordered Hopping System. I. Monte Carlo Simulation”, Phys. Rev. B, 49, 11, pp.7507-7513, 1999.

[13] R. Fitzpatrick, "Quantum Mechanics: An Intermediate Level Course", http://farside.ph.utexas.edu/teaching/qmech/lectures/lectures.html.

[14] B.N. Limketkai, and M.A. Baldo, "Charge Injection into Cathode-doped Amorphous Organic Semiconductors", Phys. Rev. B., vol. 71, pp. 085207-1-085207-9, 2005.

[15] S.V. Novikov, and G.G. Malliaras, "Roughness-induced Energetic Disorder at the Metal/Organic Interface”, Phys. Rev. B., vol. 73, pp. 033302-1-033302-4, 2006.

[16] W. Gao, and A. Kahn, "Electronic Structure and Current Injection in Zinc Phthalocyanine Doped with Tetra uorotetracyanoquinodimethane", Org. El., 3, pp. 53-63, 2002.

[17] K.A. Fichthorn, and and W.H. Weinberg, "Theoretical Foundations of Dynamical Monte Carlo Simulations", J. Chem. Phys., 95, 2, pp. 1090-1096, 1991. 